FUNCTION SPACES XII

BANACH CENTER PUBLICATIONS, VOLUME 119

INSTITUTE OF MATHEMATICS

POLISH ACADEMY OF SCIENCES

WARSZAWA 2019

\title{
DUALITY FOR LOGARITHMIC INTERPOLATION SPACES AND APPLICATIONS TO BESOV SPACES
}

\author{
FERNANDO COBOS \\ Departamento de Análisis Matemático y Matemática Aplicada \\ Facultad de Matemáticas, Universidad Complutense de Madrid \\ Plaza de Ciencias 3, 28040 Madrid, Spain \\ E-mail:cobos@mat.ucm.es
}

\section{LUZ M. FERNÁNDEZ-CABRERA}

Sección Departamental de Análisis Matemático y Matemática Aplicada

Facultad de Estudios Estadisticos, Universidad Complutense de Madrid 28040 Madrid, Spain

E-mail: luz_fernandez-c@mat.ucm.es

\begin{abstract}
We review several results on duality of logarithmic interpolation spaces and applications to Besov spaces. We also establish some new results on Besov spaces with smoothness close to zero defined by differences.
\end{abstract}

1. Introduction. Besov spaces have a central role in the theory of function spaces as it can be seen in the monographs by Triebel [33, 34, 35] on the topic. There are several characterizations of these spaces. Two of the more prominent being the characterization by differences, which leads to the spaces $\mathbf{B}_{p, q}^{s}$, and the characterization based on the Fourier transform, which generates the spaces $B_{p, q}^{s}$. We recall them in Section 2

If $s>0$ it turns out that $\mathbf{B}_{p, q}^{s}=B_{p, q}^{s}$ (see [33]), but this is not the case for $s=0$. Spaces $\mathbf{B}_{p, q}^{0}$ and $B_{p, q}^{0}$ have smoothness zero and are near $L_{p}$ but they have more properties than $L_{p}$ due to their structure of Besov spaces. To compare them it is useful to consider more general spaces $\mathbf{B}_{p, q}^{0, b}, B_{p, q}^{0, b}$, including logarithmic smoothness.

2010 Mathematics Subject Classification: Primary 46E35, 46M35; Secondary 46B70.

Key words and phrases: Besov spaces, logarithmic smoothness, duality, logarithmic interpolation spaces.

The paper is in final form and no version of it will be published elsewhere. 
Interpolation theory is a useful tool to study function spaces. To work with the spaces $\mathbf{B}_{p, q}^{0, b}, B_{p, q}^{0, b}$ it is suitable to use a logarithmic perturbation of the real interpolation method that we describe in Section 3 . Then, in Section 4, we recall the duality results established by Cobos and Segurado [17] and Besoy and Cobos [3] for this interpolation method. We also review the results of Cobos and Dominguez [8] and Besoy and Cobos [3] where the duals of the spaces $\mathbf{B}_{p, q}^{0, b}$ are determined with the help of the duality formulae for interpolation spaces and the logarithmic Lipschitz spaces $\operatorname{Lip}_{p, q}^{(1,-\alpha)}$.

We finish the paper with some new results. They are contained in Section 5 and they show another description of the dual of $\mathbf{B}_{p, q}^{0, b}$ without involving Lipschitz spaces.

2. Besov spaces. Let $f$ be a function in $L_{p}=L_{p}\left(\mathbb{R}^{n}\right), 1 \leq p \leq \infty$. For $h \in \mathbb{R}^{n}$, we put $\left(\Delta_{h}^{1} f\right)(x)=f(x+h)-f(x)$ for the first difference of $f$. The subsequent differences are defined by induction: If $k \in \mathbb{N},\left(\Delta_{h}^{k+1} f\right)(x)=\Delta_{h}^{1}\left(\Delta_{h}^{k} f\right)(x)$. The $k$-th order modulus of smoothness of $f$ is given by

$$
\omega_{k}(f, t)_{p}=\sup _{|h| \leq t}\left\|\Delta_{h}^{k} f\right\|_{L_{p}}, t>0 .
$$

We write $\omega(f, t)_{p}$ if $k=1$.

For $s \geq 0, k>s, 1 \leq p \leq \infty$ and $0<q \leq \infty$, the Besov space $\mathbf{B}_{p, q}^{s}$ on $\mathbb{R}^{n}$ consists of all $f \in L_{p}$ such that the quasi-norm

$$
\|f\|_{\mathbf{B}_{p, q}^{s}}=\|f\|_{L_{p}}+\left(\int_{0}^{1}\left[t^{-s} \omega_{k}(f, t)_{p}\right]^{q} \frac{d t}{t}\right)^{1 / q}
$$

is finite (as usual, the integral should be replaced by the supremum if $q=\infty$ ).

This definition does not depend on $k>s$.

For the following space we need a smooth dyadic resolution of unity. Take $\varphi_{0}$ in the Schwartz space $\mathcal{S}$ of rapidly decreasing infinitely differentiable functions on $\mathbb{R}^{n}$ such that

$$
\operatorname{supp} \varphi_{0} \subset\left\{x \in \mathbb{R}^{n}:|x| \leq 2\right\} \quad \text { and } \quad \varphi_{0}(x)=1 \text { if }|x| \leq 1 .
$$

For $j \in \mathbb{N}$ we put

$$
\varphi_{j}(x)=\varphi_{0}\left(2^{-j} x\right)-\varphi_{0}\left(2^{-j+1} x\right), \quad x \in \mathbb{R}^{n} .
$$

Then $\left(\varphi_{j}\right)_{j=0}^{\infty}$ forms a dyadic resolution of unity: $\sum_{j=0}^{\infty} \varphi_{j}(x)=1, x \in \mathbb{R}^{n}$.

Given $-\infty<s<\infty, 1 \leq p \leq \infty$ and $0<q \leq \infty$, the space $B_{p, q}^{s}$ is formed by all tempered distributions $f \in \mathcal{S}^{\prime}$ having a finite quasi-norm

$$
\|f\|_{B_{p, q}^{s}}=\left(\sum_{j=0}^{\infty}\left(2^{j s}\left\|\mathcal{F}^{-1}\left(\varphi_{j} \mathcal{F} f\right)\right\|_{L_{p}}\right)^{q}\right)^{1 / q} .
$$

Here $\mathcal{F} f$ denotes the Fourier transform of $f$ and $\mathcal{F}^{-1}$ the inverse Fourier transform.

If $s>0$, it turns out that these two kinds of spaces coincide, $\mathbf{B}_{p, q}^{s}=B_{p, q}^{s}$ with equivalence of quasi-norms (see [33, 2.5.12]). But this is not the case if $s=0$. To compare the spaces with smoothness zero, it is useful to consider more general spaces, including in addition to the classical smoothness $s$, logarithmic smoothness with exponent $b$. 
For $-\infty<b<\infty$ and $s, p, q$ as before, we put

$$
\begin{gathered}
B_{p, q}^{s, b}=\left\{f \in \mathcal{S}^{\prime}:\|f\|_{B_{p, q}^{s, b}}=\left(\sum_{j=0}^{\infty}\left(2^{j s}(1+j)^{b}\left\|\mathcal{F}^{-1}\left(\varphi_{j} \mathcal{F} f\right)\right\|_{L_{p}}\right)^{q}\right)^{1 / q}<\infty\right\}, \\
\mathbf{B}_{p, q}^{s, b}=\left\{f \in L_{p}:\|f\|_{\mathbf{B}_{p, q}^{s, b}}=\|f\|_{L_{p}}+\left(\int_{0}^{1}\left[t^{-s}(1-\log t)^{b} \omega_{k}(f, t)_{p}\right]^{q} \frac{d t}{t}\right)^{1 / q}<\infty\right\} .
\end{gathered}
$$

Again $\mathbf{B}_{p, q}^{s, b}=B_{p, q}^{s, b}$ if $s>0$ (see [25, Theorem 2.5]). If $s=0$, the case of interest for $\mathbf{B}_{p, q}^{0, b}$ is $-1 / q \leq b$, because otherwise

$$
\int_{0}^{1}(1-\log t)^{b q} \frac{d t}{t}<\infty \quad \text { and so } \quad \mathbf{B}_{p, q}^{0, b}=L_{p} .
$$

Improving a previous result by Triebel [36, Proposition 9], the following result was shown by Cobos and Domínguez [8, Theorem 3.3]:

TheOREM 2.1. Let $1<p<\infty, 0<q \leq \infty$ and $b>-1 / q$. Then

$$
B_{p, q}^{0, b+1 / \min \{2, p, q\}} \hookrightarrow \mathbf{B}_{p, q}^{0, b} \hookrightarrow B_{p, q}^{0, b+1 / \max \{2, p, q\}} .
$$

In particular, $\mathbf{B}_{2,2}^{0, b}=B_{2,2}^{0, b+1 / 2}$ if $b>-1 / 2$ and $\mathbf{B}_{2,2}^{0}=B_{2,2}^{0,1 / 2}$.

The extreme cases $b=-1 / q$ and $p=1$ or $\infty$ have been studied in [9]. Then there is another jump in the scale, with the result that the space $\mathbf{B}_{2,2}^{0,-1 / 2}$ does not coincide with $B_{2,2}^{0}$ but with the Besov space of Fourier type $B_{2,2}^{0,0,1 / 2}$ having smoothness of the type of an iterated logarithm to the power $1 / 2$ (see [9, Corollary 3.4 and Theorem 3.7]). For the cases $p=1$ or $\infty$, see [9, Theorems $3.9,3.10,3.11]$.

Theorem 2.1 points out that in order to compare Besov spaces defined by the modulus of smoothness with those given by the Fourier transform it is essential to work with logarithmically perturbed Besov spaces. In fact, these kind of generalized Besov spaces have received attention by a number of authors. See, for example, the papers by Leopold [27], Caetano, Gogatishvili and Opic [5], Cobos and Kühn [15], Cobos, Domínguez and Triebel [1] and the references given in them. Among other things, these spaces are useful in the study of compactness in limiting embeddings.

3. Interpolation methods. Interpolation theory is a useful tool to develop the theory of Besov spaces. In fact, the proof of Theorem 2.1 is accomplished by using a modification of the real interpolation method. Next we recall the construction of the real method.

Let $\bar{A}=\left(A_{0}, A_{1}\right)$ be a Banach couple, that is, two Banach spaces $A_{0}, A_{1}$ which are continuously embedded in a common linear Hausdorff space. So, we can form their sum $A_{0}+A_{1}$ and their intersection $A_{0} \cap A_{1}$.

Peetre's $K$ - and J-functionals are defined for $t>0$ by $K(t, a)=K\left(t, a ; A_{0}, A_{1}\right)=\inf \left\{\left\|a_{0}\right\|_{A_{0}}+t\left\|a_{1}\right\|_{A_{1}}: a=a_{0}+a_{1}, a_{j} \in A_{j}\right\}, a \in A_{0}+A_{1}$, and

$$
J(t, a)=J\left(t, a ; A_{0}, A_{1}\right)=\max \left\{\|a\|_{A_{0}}, t\|a\|_{A_{1}}\right\}, \quad a \in A_{0} \cap A_{1} .
$$

Note that $K(1, \cdot)$ and $J(1, \cdot)$ are the norms on $A_{0}+A_{1}$ and $A_{0} \cap A_{1}$, respectively. 
For $0<\theta<1$ and $0<q \leq \infty$, the real interpolation space $\left(A_{0}, A_{1}\right)_{\theta, q}$ is formed by all $a \in A_{0}+A_{1}$ having a finite quasi-norm

$$
\|a\|_{\left(A_{0}, A_{1}\right)_{\theta, q}}=\left(\int_{0}^{\infty}\left(t^{-\theta} K(t, a)\right)^{q} \frac{d t}{t}\right)^{1 / q},
$$

where again the integral should be replaced by the supremum if $q=\infty$. We refer to [2, 32, 4, 1] for full details on the real method.

For $1 \leq p \leq \infty, 0<q, q_{0}, q_{1} \leq \infty,-\infty<s_{0} \neq s_{1}<\infty$ and $0<\theta<1$, it turns out that

$$
\left(B_{p, q_{0}}^{s_{0}}, B_{p, q_{1}}^{s_{1}}\right)_{\theta, q}=B_{p, q}^{s} \quad \text { where } \quad s=(1-\theta) s_{0}+\theta s_{1} .
$$

The following interpolation result is new. It shows that logarithmic smoothness also appears by interpolation of classical spaces $\mathbf{B}_{p, q}^{0}$.

Theorem 3.1. Let $1 \leq p \leq \infty, 0<\theta<1,0<q_{0} \neq q_{1}<\infty$ and $b=(1-\theta) / q_{0}+\theta / q_{1}-$ $1 / q$. Then we have with equivalence of quasi-norms

$$
\left(\mathbf{B}_{p, q_{0}}^{0}, \mathbf{B}_{p, q_{1}}^{0}\right)_{\theta, q}=\mathbf{B}_{p, q}^{0, b}
$$

Proof. According to [11, Lemma 3.3], the spaces $\mathbf{B}_{p, q_{j}}^{0}$ are approximation spaces on $L_{p}$ when we take as approximation family the subsets

$$
G_{k}=\left\{g \in L_{p}: \operatorname{supp} \mathcal{F} g \subseteq\left\{x \in \mathbb{R}^{n}:|x| \leq k\right\}\right\}, \quad k \in \mathbb{N},
$$

and $G_{0}=\{0\}$. Namely,

$$
\mathbf{B}_{p, q_{j}}^{0}=\left\{f \in L_{p}:\|f\|=\left(\sum_{k=1}^{\infty} E_{k}(f)_{p}^{q_{j}} k^{-1}\right)^{1 / q_{j}}<\infty\right\}
$$

and $\quad \mathbf{B}_{p, q}^{0, b}=\left\{f \in L_{p}:\|f\|=\left(\sum_{k=1}^{\infty}\left[(1+\log k)^{b} E_{k}(f)_{p}\right]^{q} k^{-1}\right)^{1 / q}<\infty\right\}$

$$
\text { with } \quad E_{k}(f)_{p}=\inf \left\{\|f-g\|_{L_{p}}: g \in G_{k-1}\right\} .
$$

Consequently, using the interpolation formula for approximation spaces established by Fehér and Grässler [21, Theorem 5] (see also [10, (3.6)]) we conclude that $\left(\mathbf{B}_{p, q_{0}}^{0}, \mathbf{B}_{p, q_{1}}^{0}\right)_{\theta, q}=\mathbf{B}_{p, q}^{0, b}$.

The following logarithmic perturbation of the real method is useful to work with Besov spaces having logarithmic smoothness.

Let $\ell(t)=1+|\log t|, \ell \ell(t)=1+\log (1+|\log t|)$ and for $\mathbb{A}=\left(\alpha_{0}, \alpha_{\infty}\right) \in \mathbb{R}^{2}$ put

$$
\ell^{\mathbb{A}}(t)=\ell^{\left(\alpha_{0}, \alpha_{\infty}\right)}(t)=\left\{\begin{array}{lll}
(1+|\log t|)^{\alpha_{0}} & \text { if } & 0<t \leq 1 \\
(1+|\log t|)^{\alpha_{\infty}} & \text { if } & 1<t<\infty
\end{array}\right.
$$

and define $\ell \ell^{\mathbb{A}}(t)$ similarly.

For $0 \leq \theta \leq 1$ and $0<q \leq \infty$, we put

$$
\left(A_{0}, A_{1}\right)_{\theta, q, \mathbb{A}}=\left\{a \in A_{0}+A_{1}:\|a\|_{\left(A_{0}, A_{1}\right)_{\theta, q, \mathbb{A}}}=\left(\int_{0}^{\infty}\left(t^{-\theta} \ell^{\mathbb{A}}(t) K(t, a)\right)^{q} \frac{d t}{t}\right)^{1 / q}<\infty\right\} .
$$

If $\alpha_{0}=\alpha_{\infty}=\alpha$, we simply write $\left(A_{0}, A_{1}\right)_{\theta, q, \alpha}$. Clearly, $\left(A_{0}, A_{1}\right)_{\theta, q, 0}=\left(A_{0}, A_{1}\right)_{\theta, q}$. 
Note that we allow now that $\theta$ takes the extreme values 0 and 1 because, as we shall see later, the definition is meaningful if we ask $\mathbb{A}$ to satisfy a certain condition.

If $0<\theta<1$ then the space $\left(A_{0}, A_{1}\right)_{\theta, q, \mathbb{A}}$ is a special instance of the real method with a function parameter already studied by Gustavsson [22, Janson [26], Ovchinnikov [29] and Persson 31 among other authors. The resulting theory is close to the theory of the real method. Some results that we will show later will illustrate this fact. However, if $\theta=0$ or $\theta=1$, the function $t^{-\theta} \ell^{\mathbb{A}}(t)$ does not satisfy the assumptions of these papers. Logarithmic methods, including those extreme values, have been studied in the papers by Evans and Opic [19] and Evans, Opic and Pick [20]. However, there were some natural open questions for these methods when $\theta$ takes the extreme values 0 or 1 . Let us take a more careful look at these cases.

First note that $K\left(t, a ; A_{0}, A_{1}\right)=t K\left(t^{-1}, a ; A_{1}, A_{0}\right)$. Hence, a change of variables yields that $\left(A_{0}, A_{1}\right)_{0, q,\left(\alpha_{0}, \alpha_{\infty}\right)}=\left(A_{1}, A_{0}\right)_{1, q,\left(\alpha_{\infty}, \alpha_{0}\right)}$. Therefore, it is enough to study just one of the two spaces. Then this formula allows transferring the results to the other method. We will consider here the case $\theta=1$.

The first difference with respect to the real method is that now it may happen that $\left(A_{0}, A_{1}\right)_{1, q, \mathbb{A}}=\{0\}$. In order to avoid it, we should assume that

$$
\left\{\begin{array}{lll}
\alpha_{0}+1 / q<0 & \text { if } & q<\infty \\
\alpha_{0}<0 & \text { if } & q=\infty
\end{array}\right.
$$

Under this assumption, it turns out that

$$
A_{0} \cap A_{1} \hookrightarrow\left(A_{0}, A_{1}\right)_{1, q, \mathbb{A}} \hookrightarrow A_{0}+A_{1} .
$$

Here, $\hookrightarrow$ means continuous embedding. The interpolation property for linear operators holds for these constructions.

If $A_{0} \hookrightarrow A_{1}$ and $\|a\|_{A_{1}} \leq\|a\|_{A_{0}}$ for $a \in A_{0}$, then $K(t, a)=t\|a\|_{A_{1}}$ for $0<t \leq 1$. In this case we can disregard the part of the integral over the interval $(0,1)$ in the quasi-norm

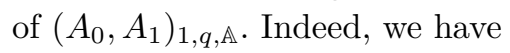

$$
\left(\int_{0}^{1}\left[t^{-1} \ell^{\alpha_{0}}(t) K(t, a)\right]^{q} \frac{d t}{t}\right)^{1 / q}=\|a\|_{A_{1}}\left(\int_{0}^{1} \ell^{\alpha_{0} q}(t) \frac{d t}{t}\right)^{1 / q} .
$$

The last integral is finite by our assumption on $\alpha_{0}$. Hence, replacing the integral by the finite integral $\left(\int_{1}^{\infty}\left[t^{-1} \ell^{\alpha}(t)\right]^{q} \frac{d t}{t}\right)^{1 / q}$, and since $K(t, a)$ is a increasing function, we get

$$
\begin{aligned}
\left(\int_{0}^{1}\left[t^{-1} \ell^{\alpha_{0}}(t) K(t, a)\right]^{q} \frac{d t}{t}\right)^{1 / q} & \leq c\|a\|_{A_{1}}\left(\int_{1}^{\infty}\left[t^{-1} \ell^{\alpha_{\infty}}(t)\right]^{q} \frac{d t}{t}\right)^{1 / q} \\
& \leq c\left(\int_{1}^{\infty}\left[t^{-1} \ell^{\alpha_{\infty}}(t) K(t, a)\right]^{q} \frac{d t}{t}\right)^{1 / q} .
\end{aligned}
$$

This shows that

$$
\|a\|_{\left(A_{0}, A_{1}\right)_{1, q, \mathbb{A}}} \sim\left(\int_{1}^{\infty}\left[\frac{K(t, a)}{t(1+\log t)^{-\alpha_{\infty}}}\right]^{q} \frac{d t}{t}\right)^{1 / q} .
$$

Hence the parameter $\alpha_{0}$ has no role when $A_{0} \hookrightarrow A_{1}$. The equivalence (1) shows the connection between logarithmic interpolation spaces and the so-called limiting interpolation spaces for ordered couples which have been studied in [13, 14, 16, 7] among other papers. 
We can now realize another important difference between logarithmic methods with $\theta=1$ and the real method. It is well-known that if $q<\infty$ then $A_{0} \cap A_{1}$ is dense in $\left(A_{0}, A_{1}\right)_{\theta, q}$ (see, for example, 2, Theorem 3.4.2/(b)]). However, this density property does not hold in general for $\left(A_{0}, A_{1}\right)_{1, q, \mathbb{A}}$. Indeed, assume that $\alpha_{\infty}$ satisfies

$$
\left\{\begin{array}{lll}
\alpha_{\infty}+1 / q<0 & \text { if } & q<\infty \\
\alpha_{\infty} \leq 0 & \text { if } & q=\infty
\end{array}\right.
$$

and that $A_{0} \hookrightarrow A_{1}$ with embedding having norm less than or equal to 1 . Since $\|a\|_{A_{1}}=$ $\|a\|_{A_{0}+A_{1}}=K(1, a)$ and the function $K(t, a) / t$ is non-increasing, we obtain

$$
\begin{aligned}
\|a\|_{\left(A_{0}, A_{1}\right)_{1, q, \mathbb{A}}} & \sim\left(\int_{1}^{\infty}\left[\frac{K(t, a)}{t(1+\log t)^{-\alpha_{\infty}}}\right]^{q} \frac{d t}{t}\right)^{1 / q} \\
& \leq\|a\|_{A_{1}}\left(\int_{1}^{\infty}(1+\log t)^{\alpha_{\infty}} \frac{d t}{t}\right)^{1 / q} \leq c\|a\|_{A_{1}} .
\end{aligned}
$$

This yields that $\left(A_{0}, A_{1}\right)_{1, q, \mathbb{A}}=A_{1}$ with equivalence of quasi-norms. In particular, $\left(\ell_{1}, \ell_{\infty}\right)_{1, q, \mathbb{A}}=\ell_{\infty}$. Since $\ell_{1}=\ell_{1} \cap \ell_{\infty}$ is not dense in $\ell_{\infty}$, this example shows that in general the intersection is not dense in the interpolation space if $\alpha_{\infty}$ satisfies (2).

Density property of the real method is a consequence of its representation in terms of the $J$-functional. For the case of logarithmic methods with $\theta=1$, the representation in terms of the $J$-functional has been studied by Cobos and Segurado [17], in the case of $1 \leq q \leq \infty$, and Besoy and Cobos [3], when $0<q<1$. Note that the example with $\left(\ell_{1}, \ell_{\infty}\right)$ that we have just seen shows that there is no $J$-representation when $\alpha_{\infty}$ is in the range 2 .

Let on the other hand

$$
\begin{cases}\alpha_{\infty} \geq 0 & \text { if } 0<q \leq 1 \\ \alpha_{\infty}-1 / q^{\prime}>0 & \text { if } 1<q \leq \infty\end{cases}
$$

where $1 / q+1 / q^{\prime}=1$ if $1 \leq q \leq \infty$. The space $\left(A_{0}, A_{1}\right)_{1, q, \mathbb{A}}^{J}$ is formed by all those $a \in A_{0}+A_{1}$ for which there exists a sequence $\left(u_{m}\right)_{m \in \mathbb{Z}} \subseteq A_{0} \cap A_{1}$ such that

$$
\left.a=\sum_{m=-\infty}^{\infty} u_{m} \quad \text { (convergence in } A_{0}+A_{1}\right)
$$

and

$$
\left(\sum_{m=-\infty}^{\infty}\left[2^{-m} \ell^{\mathbb{A}}\left(2^{m}\right) J\left(2^{m}, u_{m}\right)\right]^{q}\right)^{1 / q}<\infty .
$$

We endow $\left(A_{0}, A_{1}\right)_{1, q, \mathbb{A}}^{J}$ with the quasi-norm

$$
\|a\|_{\left(A_{0}, A_{1}\right)_{1, q, \mathbb{A}}^{J}}=\inf \left\{\left(\sum_{m=-\infty}^{\infty}\left[2^{-m} \ell^{\mathbb{A}}\left(2^{m}\right) J\left(2^{m}, u_{m}\right)\right]^{q}\right)^{1 / q}: a=\sum_{m=-\infty}^{\infty} u_{m}\right\} .
$$

If $\alpha_{\infty}+1 / q>0$ and $1 \leq q \leq \infty$, it is shown in [17, Theorem 3.5] that

$$
\left(A_{0}, A_{1}\right)_{1, q,\left(\alpha_{0}, \alpha_{\infty}\right)}=\left(A_{0}, A_{1}\right)_{1, q,\left(\alpha_{0}+1, \alpha_{\infty}+1\right)}^{J}
$$


with equivalence of norms. So, in this range, to go from the $K$-representation to the $J$-representation, one should correct the exponents of the logarithm by adding one unit. Note also that $q$ has no influence in the new exponents of the logarithm.

If $\alpha_{\infty}+1 / q>0$ and $0<q<1$, then by [3. Theorem $3.2 /(\mathrm{i})$ ] we have

$$
\left(A_{0}, A_{1}\right)_{1, q,\left(\alpha_{0}, \alpha_{\infty}\right)}=\left(A_{0}^{\sim}, A_{1}^{\sim}\right)_{1, q,\left(\alpha_{0}+1 / q, \alpha_{\infty}+1 / q\right)}^{J} .
$$

Here $A_{j}$ is the Gagliardo completion of $A_{j}$ in $A_{0}+A_{1}$ (see [1, Definition 5.1 .3 and Theorem 5.1.4]). So $A_{j} \hookrightarrow A_{j}^{\sim} \hookrightarrow A_{0}+A_{1}$. Note the difference from the previous case: now the correction in the exponents of the logarithm changes with the value of $q$.

If $\alpha_{\infty}=-1 / q$ and $1 \leq q<\infty$ (respectively, $0<q<1$ ) then the $J$-representation also exists but in addition to the changes indicated for the case $\alpha_{\infty}+1 / q>0$, an iterated logarithm to the power 1 (respectively, to the power $1 / q$ ) should be also inserted in the part of the sum on $\mathbb{N}$ (see [17, Theorem 3.6] and [3, Theorem 3.2/(ii)]).

4. Duality. With the help of those $J$-representations, the dual of $\left(A_{0}, A_{1}\right)_{1, q,\left(\alpha_{0}, \alpha_{\infty}\right)}$ has been investigated in [17, 3]. In what follows, we assume that the Banach couple $\left(A_{0}, A_{1}\right)$ is regular, that is, $A_{0} \cap A_{1}$ is dense in $A_{0}$ and $A_{1}$. So, the pair formed by the dual spaces $\left(A_{0}^{\prime}, A_{1}^{\prime}\right)$ is also a Banach couple.

As is well-known (see, for example, [32, Theorem 1.11.2]), for $1 \leq q<\infty$ and $1 / q+$ $1 / q^{\prime}=1$, the duality formula for the real method says that $\left(\left(A_{0}, A_{1}\right)_{\theta, q}\right)^{\prime}=\left(A_{0}^{\prime}, A_{1}^{\prime}\right)_{\theta, q^{\prime}}$.

If $\theta, q$ are as before and $\left(\alpha_{0}, \alpha_{\infty}\right) \in \mathbb{R}^{2}$, then it follows from a result of Cwikel and Peetre [18, Theorem 3.1] (see also [31, Theorem 2.4]), that $\left(\left(A_{0}, A_{1}\right)_{\theta, q,\left(\alpha_{0}, \alpha_{\infty}\right)}\right)^{\prime}=$ $\left(A_{0}^{\prime}, A_{1}^{\prime}\right)_{\theta, q^{\prime},\left(-\alpha_{\infty},-\alpha_{0}\right)}$.

When $\theta=1$ the dual of the logarithmic spaces, described in terms of the $K$-functional, depends again on the relationship between $q$ and $\mathbb{A}$. The following result is proved in 17 , Theorems 5.6, 5.8 and 5.10].

THEOREM 4.1. Let $\left(A_{0}, A_{1}\right)$ be a regular Banach couple. Let $1 \leq q<\infty, 1 / q+1 / q^{\prime}=1$ and $\left(\alpha_{0}, \alpha_{\infty}\right) \in \mathbb{R}^{2}$ such that $\alpha_{0}+1 / q<0$.

(a) If $\alpha_{\infty}+1 / q>0$ then $\left(\left(A_{0}, A_{1}\right)_{1, q,\left(\alpha_{0}, \alpha_{\infty}\right)}\right)^{\prime}=\left(A_{0}^{\prime}, A_{1}^{\prime}\right)_{1, q^{\prime},\left(-1-\alpha_{\infty},-1-\alpha_{0}\right)}$.

(b) If $\alpha_{\infty}=-1 / q$ then $\left(\left(A_{0}, A_{1}\right)_{1, q,\left(\alpha_{0},-1 / q\right)}\right)^{\prime}$ equals

$$
\left\{f \in A_{0}^{\prime}+A_{1}^{\prime}:\|f\|=\left(\int_{0}^{\infty}\left[\frac{\ell^{\left(-1 / q^{\prime},-1-\alpha_{0}\right)}(t) \ell \ell^{(-1,0)}(t) K(t, f)}{t}\right]^{q^{\prime}} \frac{d t}{t}\right)^{1 / q^{\prime}}<\infty\right\} .
$$

(c) If $\alpha_{\infty}+1 / q<0$ then $\left(\left(A_{0}, A_{1}\right)_{1, q,\left(\alpha_{0}, \alpha_{\infty}\right)}\right)^{\prime}=A_{1}^{\prime} \cap\left(A_{0}^{\prime}, A_{1}^{\prime}\right)_{1, q^{\prime},\left(-1-1 / q^{\prime},-1-\alpha_{0}\right)}$.

These interpolation formulae allow us to describe the dual spaces of Besov spaces for $1 \leq q<\infty$ having in mind that for the generalized Sobolev spaces $H_{p}^{s}$ with $-\infty<s<\infty$, $1<p<\infty$ and $1 / p+1 / p^{\prime}=1$, we have $\left(H_{p}^{s}\right)^{\prime}=H_{p^{\prime}}^{-s}$ (see [32, Theorem 2.6.1/(1)]). Indeed, in the classical case, if $-\infty<s<\infty, 1<p<\infty$ and $1 \leq q<\infty$, if we choose $s_{0}<s<s_{1}$ and $0<\theta<1$ with $s=(1-\theta) s_{0}+\theta s_{1}$ then $B_{p, q}^{s}=\left(H_{p}^{s_{0}}, H_{p}^{s_{1}}\right)_{\theta, q}$ and therefore

$$
\left(B_{p, q}^{s}\right)^{\prime}=\left(\left(H_{p}^{s_{0}}, H_{p}^{s_{1}}\right)_{\theta, q}\right)^{\prime}=\left(H_{p^{\prime}}^{-s_{0}}, H_{p^{\prime}}^{-s_{1}}\right)_{\theta, q^{\prime}}=B_{p^{\prime}, q^{\prime}}^{-s} .
$$

If we work with additional logarithmic smoothness $-\infty<b<\infty$, then choosing $s_{0}, s_{1}, \theta$ as before but using the logarithmic interpolation method, we have $B_{p, q}^{s, b}=\left(H_{p}^{s_{0}}, H_{p}^{s_{1}}\right)_{\theta, q, b}$ 
(see [12, Theorem 5.3]). Consequently

$$
\left(B_{p, q}^{s, b}\right)^{\prime}=\left(\left(H_{p}^{s_{0}}, H_{p}^{s_{1}}\right)_{\theta, q, b}\right)^{\prime}=\left(H_{p^{\prime}}^{-s_{0}}, H_{p^{\prime}}^{-s_{1}}\right)_{\theta, q^{\prime},-b}=B_{p^{\prime}, q^{\prime}}^{-s,-b} .
$$

In particular $\left(B_{p, q}^{0, b}\right)^{\prime}=B_{p^{\prime}, q^{\prime}}^{0,-b}$

In order to describe the dual of the Besov spaces given by differences $\mathbf{B}_{p, q}^{0, b}$, we first recall that for $1<p<\infty, 1 \leq q \leq \infty$ and $\alpha>1 / q$, the logarithmic Lipschitz space $\operatorname{Lip}_{p, q}^{(1,-\alpha)}$ consists of all functions $f \in L_{p}$ having a finite norm

$$
\|f\|_{\operatorname{Lip}_{p, q}^{(1,-\alpha)}}=\|f\|_{L_{p}}+\left(\int_{0}^{1}\left[\frac{\omega(t, f)_{p}}{t(1-\log t)^{\alpha}}\right]^{q} \frac{d t}{t}\right)^{1 / q} .
$$

We refer to [23, 24] and the references given there for properties of these spaces. Connection between Lipschitz spaces and Besov spaces is given by the embeddings

$$
B_{p, q}^{1,-\alpha+1 / \min \{2, p, q\}} \hookrightarrow \operatorname{Lip}_{p, q}^{(1,-\alpha)} \hookrightarrow B_{p, q}^{1,-\alpha+1 / \max \{2, p, q\}}
$$

(see [8, Theorem 5.2]).

Consider also the lift operator $I_{-1}$ defined by

$$
I_{-1} f=\mathcal{F}^{-1}\left(\left(1+|x|^{2}\right)^{-1 / 2} \mathcal{F} f\right) .
$$

The following result is proved in [8, Theorem 4.3].

TheOREM 4.2. Let $1<p<\infty, 1 \leq q<\infty$ and $b>-1 / q$. The space $\left(\mathbf{B}_{p, q}^{0, b}\right)^{\prime}$ consists of all tempered distributions $f \in H_{p^{\prime}}^{-1}$ such that $I_{-1} f \in \operatorname{Lip}_{p^{\prime}, q^{\prime}}^{(1,-b-1)}$ with $1 / p+1 / p^{\prime}=1=$ $1 / q+1 / q^{\prime}$. Moreover $\|f\|_{\left(\mathbf{B}_{p, q}^{0, b}\right)^{\prime}} \sim\left\|I_{-1} f\right\|_{\operatorname{Lip}_{p^{\prime}, q^{\prime}}^{(1,-b-1)}}$.

Next we consider the case $0<q<1$. Peetre [30] proved that if $\left(A_{0}, A_{1}\right)$ is a regular Banach couple, $0<\theta<1$ and $0<q<1$, then

$$
\left(\left(A_{0}, A_{1}\right)_{\theta, q}\right)^{\prime}=\left(A_{0}^{\prime}, A_{1}^{\prime}\right)_{\theta, \infty} .
$$

As a consequence, he derived that

$$
\left(B_{p, q}^{s}\right)^{\prime}=B_{p^{\prime}, \infty}^{-s} \quad \text { for } \quad 1<p<\infty, 1 / p+1 / p^{\prime}=1,0<q<1,-\infty<s<\infty .
$$

To get formula (4), Peetre observed that if $(E,\|\cdot\|)$ is any quasi-Banach space and we consider the semi-norm

$$
\|x\|^{\sharp}=\inf \left\{\sum_{\nu=1}^{n}\left\|x_{\nu}\right\|: x=\sum_{\nu=1}^{n} x_{\nu}, n \in \mathbb{N}\right\}
$$

and put $N=\left\{x \in E:\|x\|^{\sharp}=0\right\}$, then the completion of the quotient space $E^{\sharp}=\widehat{E / N}$ satisfies $\left(E^{\sharp}\right)^{\prime}=E^{\prime}$. Then he proved that $\left(\left(A_{0}, A_{1}\right)_{\theta, q}\right)^{\sharp}=\left(A_{0}, A_{1}\right)_{\theta, 1}$ which yields 4 having in mind the duality formula for $q=1$.

As for logarithmic methods with $0<\theta<1$ and $0<q<1$, it was established in [6], Theorem 3.1] (see also [28, Corollary 2]) that $\left(\left(A_{0}, A_{1}\right)_{\theta, q,\left(\alpha_{0}, \alpha_{\infty}\right)}\right)^{\sharp}=\left(A_{0}, A_{1}\right)_{\theta, 1,\left(\alpha_{0}, \alpha_{\infty}\right)}$. Combining it with the duality formula for $0<\theta<1$ and the interpolation properties of Besov spaces with logarithmic smoothness, one can derive that for $1<p<\infty, 1 / p+$ $1 / p^{\prime}=1,0<q<1$ and $-\infty<s, b<\infty$, we have $\left(B_{p, q}^{s, b}\right)^{\prime}=B_{p^{\prime}, \infty}^{-s,-b}$. In particular, $\left(B_{p, q}^{0, b}\right)^{\prime}=B_{p^{\prime}, \infty}^{0,-b}$ 
The dual of the space $\mathbf{B}_{p, q}^{0, b}$ for $0<q<1$ has been described in [3]. We first compute $\left(\left(A_{0}, A_{1}\right)_{1, q,\left(\alpha_{0}, \alpha_{\infty}\right)}\right)^{\#}$ when $\alpha_{\infty}+1 / q>0$. Using the $J$-descriptions and proceeding as in [30] we obtain

$$
\begin{aligned}
\left(\left(A_{0}, A_{1}\right)_{1, q,\left(\alpha_{0}, \alpha_{\infty}\right)}\right)^{\sharp} & =\left(\left(A_{0}^{\sim}, A_{1}^{\sim}\right)_{1, q,\left(\alpha_{0}+1 / q, \alpha_{\infty}+1 / q\right)}^{J}\right)^{\sharp} \\
& =\left(A_{0}^{\sim}, A_{1}^{\sim}\right)_{1,1,\left(\alpha_{0}+1 / q, \alpha_{\infty}+1 / q\right)}^{J} \\
& =\left(A_{0}^{\sim}, A_{1}^{\sim}\right)_{1,1,\left(\alpha_{0}+1 / q-1, \alpha_{\infty}+1 / q-1\right)} \\
& =\left(A_{0}, A_{1}\right)_{1,1,\left(\alpha_{0}+1 / q-1, \alpha_{\infty}+1 / q-1\right)}
\end{aligned}
$$

where the last equality follows from the fact that

$$
K\left(t, a ; A_{0}, A_{1}\right)=K\left(t, a ; A_{0}^{\sim}, A_{1}^{\sim}\right), t>0, a \in A_{0}+A_{1},
$$

(see [1, Theorem 5.1.5]). From this result and the duality formula for $q=1$ we derive the following result.

Corollary 4.3. Let $\left(A_{0}, A_{1}\right)$ be a regular Banach couple. Let $\left(\alpha_{0}, \alpha_{\infty}\right) \in \mathbb{R}^{2}$ and $0<q<1$ such that $\alpha_{0}+1 / q<0$ and $\alpha_{\infty}+1 / q>0$. Then

$$
\left(\left(A_{0}, A_{1}\right)_{1, q,\left(\alpha_{0}, \alpha_{\infty}\right)}\right)^{\prime}=\left(A_{0}^{\prime}, A_{1}^{\prime}\right)_{1, \infty,\left(-\alpha_{\infty}-1 / q,-\alpha_{0}-1 / q\right)} .
$$

As a consequence, we get in [3, Theorem 5.2] the following.

THEOREM 4.4. Let $1<p<\infty, 1 / p+1 / p^{\prime}=1,0<q<1$ and $b>-1 / q$. The space $\left(\mathbf{B}_{p, q}^{0, b}\right)^{\prime}$ is formed by all $f \in H_{p^{\prime}}^{-1}$ such that $I_{-1} f \in \operatorname{Lip}_{p^{\prime}, \infty}^{(1,-b-1 / q)}$ with $1 / p+1 / p^{\prime}=1$. Moreover

$$
\|f\|_{\left(\mathbf{B}_{p, q}^{0, b}\right)^{\prime}} \sim\left\|I_{-1} f\right\|_{\operatorname{Lip}_{p^{\prime}, \infty}^{(1,-b-1 / q)}}=\left\|I_{-1} f\right\|_{L_{p^{\prime}}}+\sup _{0<t<1}\left(\frac{\omega\left(t, I_{-1} f\right)_{p^{\prime}}}{t(1-\log t)^{b+1 / q}}\right) .
$$

In conclusion, for Besov spaces defined by the Fourier transform with classical smoothness 0 and logarithmic smoothness with exponent $b \in \mathbb{R}$ and $1<p<\infty$, we have

$$
\left(B_{p, q}^{0, b}\right)^{\prime}= \begin{cases}B_{p^{\prime}, q^{\prime}}^{0,-b} & \text { if } \quad 1 \leq q<\infty, 1 / q+1 / q^{\prime}=1=1 / p+1 / p^{\prime}, \\ B_{p^{\prime}, \infty}^{0,-b} & \text { if } \quad 0<q<1 .\end{cases}
$$

Note that the dual for $0<q<1$ is always the same space. However this is not the case for Besov spaces defined by differences where

$$
\|f\|_{\left(\mathbf{B}_{p, q}^{0, b}\right)^{\prime}} \sim \begin{cases}\left\|I_{-1} f\right\|_{\operatorname{Lip}_{p^{\prime}, q^{\prime}}^{(1,-b-1)}} & \text { if } \quad 1 \leq q<\infty \\ \left\|I_{-1} f\right\|_{\operatorname{Lip}_{p^{\prime}, \infty}^{(1,-b-1 / q)}} & \text { if } \quad 0<q<1 .\end{cases}
$$

Observe also that in the description of the dual of $\mathbf{B}_{p, q}^{0, b}$, the parameter $q$ has no role in the exponent of the associated Lipschitz space if $1 \leq q<\infty$, while if $0<q<1$ the exponent is $-b-1 / q$.

5. Another description for the dual of $\mathbf{B}_{p, q}^{0, b}$. The results of this last section are new and show another description for the dual of $\mathbf{B}_{p, q}^{0, b}$ without involving Lipschitz spaces. We start with an auxiliary result.

We put $\mathbb{Z}^{-}=\{0,-1,-2, \ldots\}$. Let $0<q \leq \infty, \alpha \in \mathbb{R}$ and let $\left(A_{0}, A_{1}\right)$ be a Banach couple. The space $\left[A_{0}, A_{1}\right]_{1, q, \alpha}^{J}$ is formed by all those $a \in A_{0}+A_{1}$ such that there exists 
$\left(w_{m}\right)_{m \in \mathbb{Z}^{-}} \subseteq A_{0} \cap A_{1}$ with $a=\sum_{m=-\infty}^{0} w_{m}$ (convergence in $A_{0}+A_{1}$ ) and

$$
\left(\sum_{m=-\infty}^{0}\left[2^{-m} \ell^{\alpha}\left(2^{m}\right) J\left(2^{m}, w_{m}\right)\right]^{q}\right)^{1 / q}<\infty .
$$

The quasi-norm in $\left[A_{0}, A_{1}\right]_{1, q, \alpha}^{J}$ is given by

$$
\|a\|_{\left[A_{0}, A_{1}\right]_{1, q, \alpha}^{J}}=\inf \left\{\left(\sum_{m=-\infty}^{0}\left[2^{-m} \ell^{\alpha}\left(2^{m}\right) J\left(2^{m}, w_{m}\right)\right]^{q}\right)^{1 / q}: a=\sum_{m=-\infty}^{0} w_{m}\right\} .
$$

Lemma 5.1. Let $A_{0}, A_{1}$ be Banach spaces such that $A_{1} \hookrightarrow A_{0}$ with the norm of the embedding being less than or equal to 1 . Let $0<q \leq \infty$ and $\mathbb{A}=\left(\alpha_{0}, \alpha_{\infty}\right) \in \mathbb{R}^{2}$ satisfying

$$
\left\{\begin{array}{ll}
\alpha_{\infty} \geq 0 & \text { if } 0<q \leq 1, \\
\alpha_{\infty}-1 / q^{\prime}>0 & \text { if } 1<q \leq \infty
\end{array} \text { and } 1 / q+1 / q^{\prime}=1 .\right.
$$

Then we have with equivalence of quasi-norms $\left(A_{0}, A_{1}\right)_{1, q, \mathbb{A}}^{J}=\left[A_{0}, A_{1}\right]_{1, q, \alpha_{0}}^{J}$.

Proof. Assume that $a \in\left(A_{0}, A_{1}\right)_{1, q, \mathbb{A}}^{J}$ and let $a=\sum_{m=-\infty}^{\infty} u_{m}$ be a representation of $a$ with

$$
\left(\sum_{m=-\infty}^{\infty}\left[2^{-m} \ell^{\mathbb{A}}\left(2^{m}\right) J\left(2^{m}, u_{m}\right)\right]^{q}\right)^{1 / q} \leq 2\|a\|_{\left(A_{0}, A_{1}\right)_{1, q, \mathbb{A}}^{J}} .
$$

Then $\sum_{m=1}^{\infty} u_{m}$ belongs to $A_{1}$ because, if $1 \leq q \leq \infty$, by Hölder's inequality we get

$$
\begin{aligned}
\sum_{m=1}^{\infty}\left\|u_{m}\right\|_{A_{1}} & \leq \sum_{m=1}^{\infty} 2^{-m} J\left(2^{m}, u_{m}\right) \\
& \leq\left(\sum_{m=1}^{\infty}\left[2^{-m} \ell^{\alpha_{\infty}}\left(2^{m}\right) J\left(2^{m}, u_{m}\right)\right]^{q}\right)^{1 / q}\left(\sum_{m=1}^{\infty} \ell^{-\alpha_{\infty} q^{\prime}}\left(2^{m}\right)\right)^{1 / q^{\prime}}
\end{aligned}
$$

and the last sum is finite due to $\alpha_{\infty}-1 / q^{\prime}>0$. If $0<q<1$, using the Jensen inequality we obtain

$$
\begin{aligned}
\sum_{m=1}^{\infty}\left\|u_{m}\right\|_{A_{1}} & \leq \sum_{m=1}^{\infty} 2^{-m} J\left(2^{m}, u_{m}\right) \leq\left(\sum_{m=1}^{\infty}\left[2^{-m} J\left(2^{m}, u_{m}\right)\right]^{q}\right)^{1 / q} \\
& \leq\left(\sum_{m=1}^{\infty}\left[2^{-m} \ell^{\alpha_{\infty}}\left(2^{m}\right) J\left(2^{m}, u_{m}\right)\right]^{q}\right)^{1 / q} \times \sup _{m \geq 1}\left\{\ell^{-\alpha_{\infty}}\left(2^{m}\right)\right\}
\end{aligned}
$$

and the supremum is also finite because now $\alpha_{\infty} \geq 0$. Let $v=\sum_{m=1}^{\infty} u_{m}$. By the previous computations we have $\|v\|_{A_{1}} \leq c_{1}\|a\|_{\left(A_{0}, A_{1}\right)_{1, q, \mathbb{A}}^{J}}$. Let $w_{m}=u_{m}$ if $m=-1,-2,-3, \ldots$ and $w_{0}=u_{0}+v$. Then $a=\sum_{m=-\infty}^{0} w_{m}$ in $A_{0}+A_{1}=A_{0}$ and

$$
\begin{aligned}
\|a\|_{\left[A_{0}, A_{1}\right]_{1, q, \alpha_{0}}^{J}} & \leq\left(\sum_{m=-\infty}^{0}\left[2^{-m} \ell^{\alpha_{0}}\left(2^{m}\right) J\left(2^{m}, w_{m}\right)\right]^{q}\right)^{1 / q} \\
& \leq c_{2}\left[\|v\|_{A_{1}}+\left(\sum_{m=-\infty}^{0}\left[2^{-m} \ell^{\alpha_{0}}\left(2^{m}\right) J\left(2^{m}, w_{m}\right)\right]^{q}\right)^{1 / q}\right] \leq c_{3}\|a\|_{\left(A_{0}, A_{1}\right)_{1, q, \mathrm{~A}}^{J}} .
\end{aligned}
$$

Hence, $\left(A_{0}, A_{1}\right)_{1, q, \mathbb{A}}^{J} \hookrightarrow\left[A_{0}, A_{1}\right]_{1, q, \alpha_{0}}^{J}$. The converse embedding is clear. 
Theorem 5.2. Let $1<p<\infty, 1 \leq q<\infty, 1 / p+1 / p^{\prime}=1=1 / q+1 / q^{\prime}$ and $b>-1 / q$. The space $\left(\mathbf{B}_{p, q}^{0, b}\right)^{\prime}$ consists of all tempered distributions $f$ such that there is a sequence $\left(f_{m}\right)_{m \in \mathbb{Z}^{-}} \subseteq L_{p^{\prime}}$ with $f=\sum_{m=-\infty}^{0} f_{m}$ (convergence in $\left.\mathcal{S}^{\prime}\right)$ and

$$
\left(\sum_{m=-\infty}^{0}\left[2^{-m} \ell^{-b}\left(2^{m}\right)\left(\left\|I_{-1} f_{m}\right\|_{L_{p^{\prime}}}+2^{m}\left\|f_{m}\right\|_{L_{p^{\prime}}}\right)\right]^{q^{\prime}}\right)^{1 / q^{\prime}}<\infty
$$

Furthermore,

$$
\|f\|_{\left(\mathbf{B}_{p, q}^{0, b}\right)^{\prime}} \sim \inf _{f=\sum_{m=-\infty}^{0} f_{m}}\left\{\left(\sum_{m=-\infty}^{0}\left[2^{-m} \ell^{-b}\left(2^{m}\right)\left(\left\|I_{-1} f_{m}\right\|_{L_{p^{\prime}}}+2^{m}\left\|f_{m}\right\|_{L_{p^{\prime}}}\right)\right]^{q^{\prime}}\right)^{1 / q^{\prime}}\right\} .
$$

Proof. Consider the couple $\left(L_{p}, W_{p}^{1}\right)$ formed by $L_{p}$ and the Sobolev space $W_{p}^{1}$. According to [1, Theorem 5.4.12], there are positive constants $c_{1}, c_{2}$ such that for any $g \in L_{p}$ and any $t>0$ we have

$$
c_{1} K\left(t, g ; L_{p}, W_{p}^{1}\right) \leq \min \{1, t\}\|g\|_{L_{p}}+\omega(g, t)_{p} \leq c_{2} K\left(t, g ; L_{p}, W_{p}^{1}\right) .
$$

Reversing the couple we get

$$
K\left(t, g ; W_{p}^{1}, L_{p}\right) \sim \min \{1, t\}\|g\|_{L_{p}}+t \omega\left(g, t^{-1}\right)_{p} .
$$

Take any $\tau$ with $\tau+1 / q<0$. It follows from the previous estimate for the $K$-functional that

$$
\mathbf{B}_{p, q}^{0, b}=\left(W_{p}^{1}, L_{p}\right)_{1, q,(\tau, b)} .
$$

Now, using Theorem 4.1/(a) and (3), we derive that

$$
\left(\mathbf{B}_{p, q}^{0, b}\right)^{\prime}=\left(H_{p^{\prime}}^{-1}, L_{p^{\prime}}\right)_{1, q^{\prime},(-b-1,-\tau-1)}=\left(H_{p^{\prime}}^{-1}, L_{p^{\prime}}\right)_{1, q^{\prime},(-b,-\tau)}^{J}=\left[H_{p^{\prime}}^{-1}, L_{p^{\prime}}\right]_{1, q^{\prime},-b}^{J}
$$

where the last equality follows from Lemma 5.1. Therefore, the definition of the $J$-space $\left[H_{p^{\prime}}^{-1}, L_{p^{\prime}}\right]_{1, q^{\prime},-b}^{J}$ shows that a tempered distribution $f$ belongs to $\left(\mathbf{B}_{p, q}^{0, b}\right)^{\prime}$ if and only if there is a sequence of functions $\left(f_{m}\right)_{m \in \mathbb{Z}^{-}} \subseteq L_{p^{\prime}}$ such that $f=\sum_{m=-\infty}^{0} f_{m}$ (convergence in $\mathcal{S}^{\prime}$ ) and

$$
\left(\sum_{m=-\infty}^{0}\left[2^{-m} \ell^{-b}\left(2^{m}\right) J\left(2^{m}, f_{m} ; H_{p^{\prime}}^{-1}, L_{p^{\prime}}\right)\right]^{q^{\prime}}\right)^{1 / q^{\prime}}<\infty
$$

Finally, since the lift $I_{-1}: H_{p^{\prime}}^{-1} \longrightarrow L_{p^{\prime}}$ is bijective and bounded, we get

$$
\|f\|_{\left(\mathbf{B}_{p, q}^{0, b}\right)^{\prime}} \sim \inf _{f=\sum_{m=-\infty}^{0} f_{m}}\left\{\left(\sum_{m=-\infty}^{0}\left[2^{-m} \ell^{-b}\left(2^{m}\right)\left(\left\|I_{-1} f_{m}\right\|_{L_{p^{\prime}}}+2^{m}\left\|f_{m}\right\|_{L_{p^{\prime}}}\right)\right]^{q^{\prime}}\right)^{1 / q^{\prime}}\right\}
$$

We finish the paper with the corresponding result for $0<q<1$.

TheOREM 5.3. Let $1<p<\infty, 1 / p+1 / p^{\prime}=1,0<q<1$ and $b>-1 / q$. The space $\left(\mathbf{B}_{p, q}^{0, b}\right)^{\prime}$ consists of all tempered distributions $f$ such that there is a sequence $\left(f_{m}\right)_{m \in \mathbb{Z}^{-}} \subseteq$ $L_{p^{\prime}}$ with $f=\sum_{m=-\infty}^{0} f_{m}$ (convergence in $\left.\mathcal{S}^{\prime}\right)$ and

$$
\sup _{m \in \mathbb{Z}^{-}}\left(2^{-m} \ell^{-b+1-1 / q}\left(2^{m}\right)\left(\left\|I_{-1} f_{m}\right\|_{L_{p^{\prime}}}+2^{m}\left\|f_{m}\right\|_{L_{p^{\prime}}}\right)\right)<\infty .
$$


Furthermore,

$$
\|f\|_{\left(\mathbf{B}_{p, q}^{0, b}\right)^{\prime}} \sim \inf _{f=\sum_{m=-\infty}^{0} f_{m}}\left\{\sup _{m \in \mathbb{Z}^{-}}\left(2^{-m} \ell^{-b+1-1 / q}\left(2^{m}\right)\left(\left\|I_{-1} f_{m}\right\|_{L_{p^{\prime}}}+2^{m}\left\|f_{m}\right\|_{L_{p^{\prime}}}\right)\right)\right\} .
$$

Proof. Proceeding as at the beginning of the proof of Theorem 5.2 we get $\mathbf{B}_{p, q}^{0, b}=$ $\left(W_{p}^{1}, L_{p}\right)_{1, q,(\tau, b)}$ with $\tau+1 / q<0$. Hence, by Corollary 4.3 , we get

$$
\left(\mathbf{B}_{p, q}^{0, b}\right)^{\prime}=\left(H_{p^{\prime}}^{-1}, L_{p^{\prime}}\right)_{1, \infty,(-b-1 / q,-\tau-1 / q)} .
$$

Now, applying (3) and Lemma 5.1, we derive that

$$
\left(\mathbf{B}_{p, q}^{0, b}\right)^{\prime}=\left(H_{p^{\prime}}^{-1}, L_{p^{\prime}}\right)_{1, \infty,(-b+1-1 / q,-\tau+1-1 / q)}^{J}=\left[H_{p^{\prime}}^{-1}, L_{p^{\prime}}\right]_{1, \infty,-b+1-1 / q}^{J} .
$$

This equality and the properties of the lift $I_{-1}$ yield that a tempered distribution $f$ belongs to $\left(\mathbf{B}_{p, q}^{0, b}\right)^{\prime}$ if and only if there exists $\left(f_{m}\right)_{m \in \mathbb{Z}^{-}} \subseteq L_{p^{\prime}}$ such that $f=\sum_{m=-\infty}^{0} f_{m}$ (convergence in $\mathcal{S}^{\prime}$ ) and

$$
\sup _{m \in \mathbb{Z}^{-}}\left(2^{-m} \ell^{-b+1-1 / q}\left(2^{m}\right)\left(\left\|I_{-1} f_{m}\right\|_{L_{p^{\prime}}}+2^{m}\left\|f_{m}\right\|_{L_{p^{\prime}}}\right)\right)<\infty .
$$

Moreover,

$$
\|f\|_{\left(\mathbf{B}_{p, q}^{0, b}\right)^{\prime}} \sim \inf _{f=\sum_{m=-\infty}^{0} f_{m}}\left\{\sup _{m \in \mathbb{Z}^{-}}\left(2^{-m} \ell^{-b+1-1 / q}\left(2^{m}\right)\left(\left\|I_{-1} f_{m}\right\|_{L_{p^{\prime}}}+2^{m}\left\|f_{m}\right\|_{L_{p^{\prime}}}\right)\right)\right\} .
$$

Acknowledgments. The authors have been supported in part by MTM2017-84058-P (AEI/FEDER, UE).

The authors would like to thank Prof. Hans Triebel for some stimulating mails and to the referee for his/her comments.

\section{References}

[1] C. Bennett, R. Sharpley, Interpolation of Operators, Pure Appl. Math. 129, Academic Press, Boston, 1988.

[2] J. Bergh, J. Löfström, Interpolation Spaces. An Introduction, Grundlehren Math. Wiss. 223, Springer, Berlin, 1976.

[3] B. F. Besoy, F. Cobos, Duality for logarithmic interpolation spaces when $0<q<1$ and applications, J. Math. Anal. Appl. 466 (2018), 373-399.

[4] Y. Brudnyı̆, N. Krugljak, Interpolation Functors and Interpolation Spaces, Vol. 1, NorthHolland Math. Library 47, North-Holland, Amsterdam, 1991.

[5] A. M. Caetano, A. Gogatishvili, B. Opic, Sharp embeddings of Besov spaces involving only logarithmic smoothness, J. Approx. Theory 152 (2008), 188-214.

[6] F. Cobos, Duality and Lorentz-Marcinkiewicz operator spaces, Math. Scand. 63 (1988), 261-267.

[7] F. Cobos, O. Domínguez, Approximation spaces, limiting interpolation and Besov spaces, J. Approx. Theory 189 (2015), 43-66.

[8] F. Cobos, O. Domínguez, On Besov spaces of logarithmic smoothness and Lipschitz spaces, J. Math. Anal. Appl. 425 (2015), 71-84.

[9] F. Cobos, O. Domínguez, On the relationship between two kinds of Besov spaces with smoothness near zero and some other applications of limiting interpolation, J. Fourier Anal. Appl. 22 (2016), 1174-1191. 
[10] F. Cobos, O. Domínguez, T. Kühn, Approximation and entropy numbers of embeddings between approximation spaces, Constr. Approx. 47 (2018), 453-486.

[11] F. Cobos, O. Domínguez, H. Triebel, Characterizations of logarithmic Besov spaces in terms of differences, Fourier-analytical decompositions, wavelets and semi-groups, J. Funct. Anal. 270 (2016), 4386-4425.

[12] F. Cobos, D. L. Fernandez, Hardy-Sobolev spaces and Besov spaces with a function parameter, in: Function Spaces and Applications, Lecture Notes in Math. 1302, Springer, Berlin, 1988, 158-170.

[13] F. Cobos, L. M. Fernández-Cabrera, T. Kühn, T. Ullrich, On an extreme class of real interpolation spaces, J. Funct. Anal. 256 (2009), 2321-2366.

[14] F. Cobos, L. M. Fernández-Cabrera, M. Mastyło, Abstract limit J-spaces, J. Lond. Math. Soc. (2) 82 (2010), 501-525.

[15] F. Cobos, T. Kühn, Approximation and entropy numbers in Besov spaces of generalized smoothness, J. Approx. Theory 160 (2009), 56-70.

[16] F. Cobos, T. Kühn, Equivalence of $K$ - and J-methods for limiting real interpolation spaces, J. Funct. Anal. 261 (2011), 3696-3722.

[17] F. Cobos, A. Segurado, Description of logarithmic interpolation spaces by means of the J-functional and applications, J. Funct. Anal. 268 (2015), 2906-2945.

[18] M. Cwikel, J. Peetre, Abstract K and J spaces, J. Math. Pures Appl. (9) 60 (1981), 1-50.

[19] W. D. Evans, B. Opic, Real interpolation with logarithmic functors and reiteration, Canad. J. Math. 52 (2000), 920-960.

[20] W. D. Evans, B. Opic, L. Pick, Real interpolation with logarithmic functors, J. Inequal. Appl. 7 (2002), 187-269.

[21] F. Fehér, G. Grässler, On an extremal scale of approximation spaces, J. Comput. Anal. Appl. 3 (2001), 95-108.

[22] J. Gustavsson, A function parameter in connection with interpolation of Banach spaces, Math. Scand. 42 (1978), 289-305.

[23] D. D. Haroske, On more general Lipschitz spaces, Z. Anal. Anwendungen 19 (2000), 781799.

[24] D. D. Haroske, Envelopes and Sharp Embeddings of Function Spaces, Chapman \& Hall/CRC Res. Notes Math. 437, Chapman \& Hall/CRC, Boca Raton, FL, 2007.

[25] D. D. Haroske, S. D. Moura, Continuity envelopes of spaces of generalized smoothness, entropy and approximation numbers, J. Approx. Theory 128 (2004), 151-174.

[26] S. Janson, Minimal and maximal methods of interpolation, J. Functional Analysis 44 (1981), $50-73$.

[27] H.-G. Leopold, Embeddings and entropy numbers in Besov spaces of generalized smoothness, in: Function Spaces, Lecture Notes in Pure and Appl. Math. 128, Dekker, New York, 2000, 323-336.

[28] M. Mastyło, Banach envelopes of some interpolation quasi-Banach spaces, in: Function Spaces and Applications, Lecture Notes in Math. 1302, Springer, Berlin, 1988, 321-329.

[29] V. I. Ovchinnikov, The method of orbits in interpolation theory, Math. Rep. 1 (1984), 349-515.

[30] J. Peetre, Remark on the dual of an interpolation space, Math. Scand. 34 (1974), 124-128.

[31] L.-E. Persson, Interpolation with a parameter function, Math. Scand. 59 (1986), 199-222.

[32] H. Triebel, Interpolation Theory, Function Spaces, Differential Operators, North-Holland Math. Library 18, North-Holland, Amsterdam, 1978. 
[33] H. Triebel, Theory of Function Spaces, Monogr. Math. 78, Birkhäuser, Basel, 1983.

[34] H. Triebel, Theory of Function Spaces II, Monogr. Math. 84, Birkhäuser, Basel, 1992.

[35] H. Triebel, Theory of Function Spaces III, Monogr. Math. 100, Birkhäuser, Basel, 2006.

[36] H. Triebel, Comments on tractable embeddings and function spaces of smoothness near zero, report, Jena, 2012. 